\title{
Study of rapid ionisation for simulation of soft X-ray lasers with the 2D hydro-radiative code ARWEN
}

\author{
F. de Gaufridy ${ }^{a, b}, *$, P. Velarde ${ }^{a, b}$, D. Portillo ${ }^{a}$, E. Oliva ${ }^{a, b}$, C. Garcia ${ }^{a}$, M. Cotelo ${ }^{a}$, A.G. de la Varga ${ }^{a}$, \\ P. Zeitoun ${ }^{\text {b }}$, S. Kazamias ${ }^{c}, K$. Cassou ${ }^{c}$, O. Guilbaud ${ }^{\text {c }}$
}

a Instituto de Fusión Nuclear, José Gutiérrez Abascal 2, 28006 Madrid, Spain

bLaboratoire d'Optique Apliquée, Chemin de la Humiére, 91671 Palaiseau, France

${ }^{\mathrm{C}}$ Laboratoire de Physique des Gaz et des Plasmas, Université Paris XI, 91 Orsay, France

Keywords:

Transient ionisation

Soft X-ray laser

NLTE emissivity

\section{A B S T R A C T}

We present our fast ionisation routine used to study transient soft X-ray lasers with ARWEN, a twodimensional hydrodynamic code incorporating adaptative mesh refinement (AMR) and radiative transport.

We compute global rates between ion stages assuming an effective temperature between singlyexcited levels of each ion. A two-step method is used to obtain in a straightforward manner the variation of ion populations over long hydrodynamic time steps. We compare our model with existing theoretical results both stationary and transient, finding that the discrepancies are moderate except for large densities. We simulate an existing Molybdenum Ni-like transient soft X-ray laser with ARWEN. Use of the fast ionisation routine leads to a larger increase in temperature and a larger gain zone than when LTE datatables are used.

\section{Introduction}

The appearance of picosecond and sub-picosecond lasers in the last twenty years has opened the way for the development of transient soft X-ray lasers and has dramatically increased the number of situations where the ionisation can not be considered as stationary. Transient soft X-ray lasers are still being developed throughout the world both for plasma diagnosis and as a possible alternative to costly synchrotron facilities for biological or material applications. Fast ionisation is the process used to obtain very high gains and saturation over short plasma lengths with limited laserpump energy and replace the large stationary soft X-ray lasers facilities.

The simulation of soft X-ray lasers is usually based on hydrodynamic codes initially dedicated to laser-plasma interactions such as in Inertial Confinement Fusion (ICF), XUV sources and astrophysics [1,2]. Due to computational limitations, much work has been constrained to one-dimension or extrapolated to 1.5 dimension assuming symmetries in plasma shape and velocity
$[3,4]$. The ARWEN code [5] is a two-dimensional hydrodynamic code with radiation transport using adaptative mesh refinement to reduce the number of cells while keeping a constant accuracy even in zones with high density, velocity and radiative or temperature gradients. This allows to have access to lateral inhomogeneities and edge effects that are particularly important for the description of soft X-ray laser refraction inside the lasing plasma [6,7].

However, the ARWEN code uses Local Thermodynamic Equilibrium (LTE) datatables for computing both opacities and ionisations [8,9]. Instantaneous ionisation prevents simulation of transient soft X-ray lasers where the slow ionisation is the requirement of keeping high lasing ion population at high temperature. The high temperature is required to maximise the population inversion of the lasing levels. For this reason we need a fast inline ionisation routine. This requirement is not limited to soft X-ray lasers, as most experiments using short pulse lasers to create plasmas lead transient NLTE conditions [10].

Many models exist for simulating nonequilibrium and/or transient plasmas and have been presented in NLTE dedicated workshops [11-14] Some models rely on very detailed atomic physics but are very time consuming [15], others rely on coarser representations but allow faster computation [16]. Nevertheless 
a convergence of steady-state ionisation and global rates from these various models has been seen over the years [17]. Concerning transient ionisation, the available results are limited because the detailed simulations are difficult.

The EHYBRID 1.5D hydrodynamic radiative code, written by G. Pert [18], is widely used in the soft X-ray laser community and includes a transient ionisation routine coupled with the hydrodynamics and the radiation transfer. This routine relies on an increasingly detailed atomic kinetics representation when the ionisation approaches the lasing ionisation stage [19]. This model is fast and stable but difficult to extract and include in another hydrodynamic code. We therefore develop a new routine, based on some of the stable algorithms developed by Pert with a coarser representation of the lasing ion.

\section{Description of the model}

In order to allow large 2D hydrodynamics computations including tens of thousands of cells without tremendously increasing the computing time, the atomic physics model has to avoid the solving of large matrices. In addition, to limit memory requirements, the number of intermediate atomic levels considered in each cell has to be constrained. Finally, the numerical scheme has to be stable and robust as thermodynamic conditions may vary by many orders of magnitude during a complete simulation.

\subsection{Ionisation}

The Colombant and Tonon model [20] including only the ground level of each ion gives straightforward access to the steady-state ionisation without the need to solve the Collisional Radiative matrix. The method takes into account only collisional ionisation, three-body and radiative recombination through semi-empirical formulae. It remains widely used, for example in the EUV community [21-23], as it leads to reasonable ionisation balance compared to more refined models. At very low density, excited levels are weakly populated and their contribution to ionisation and recombination rates is very limited - except for radiative recombination. Therefore, the global rates between ions in this model remain comparable to those of very detailed models. But when the density increases, discrepancies of many orders of magnitude appear between Colombant and Tonon rates and those obtained in the NLTE workshop databases. Due to the lack of excited levels, many ionisation or recombination paths are closed. This does not affect the steady-state ionisation because semi-empirical radiative recombination rates include recombination into fictitious excited states and the collisional ionisation and three-body recombination are in balance. However, the time-dependent ionisation and recombination is strongly slowed. This can be observed in Fig. 1.

In order to include some excited levels without increasing the number of Collisional Radiative equations, we use an effective temperature law. We assume that each excited level is in steadystate with the next ion ground level [24]. Using Van Regemorter formula for collisional excitation the populations of the excited levels only depend on their energies so we can write:

$N_{i}^{Z} \approx \frac{g_{i}}{g_{0}} N_{0}^{Z} \frac{e^{-\Delta E_{i 0} / T_{e}}}{1+1.310^{13} \Delta E_{i 0}^{3} \frac{\sqrt{T_{e}}}{N_{e}}}$

Then the population of the ion writes:

$N^{Z}=N_{0}\left(1+\sum_{i>0} \frac{g_{i}}{g_{0}} \frac{e^{-\Delta E_{i 0} / T_{e}}}{1+1.310^{13} \Delta E_{i 0}^{3} \frac{\sqrt{T_{e}}}{N_{e}}}\right)$

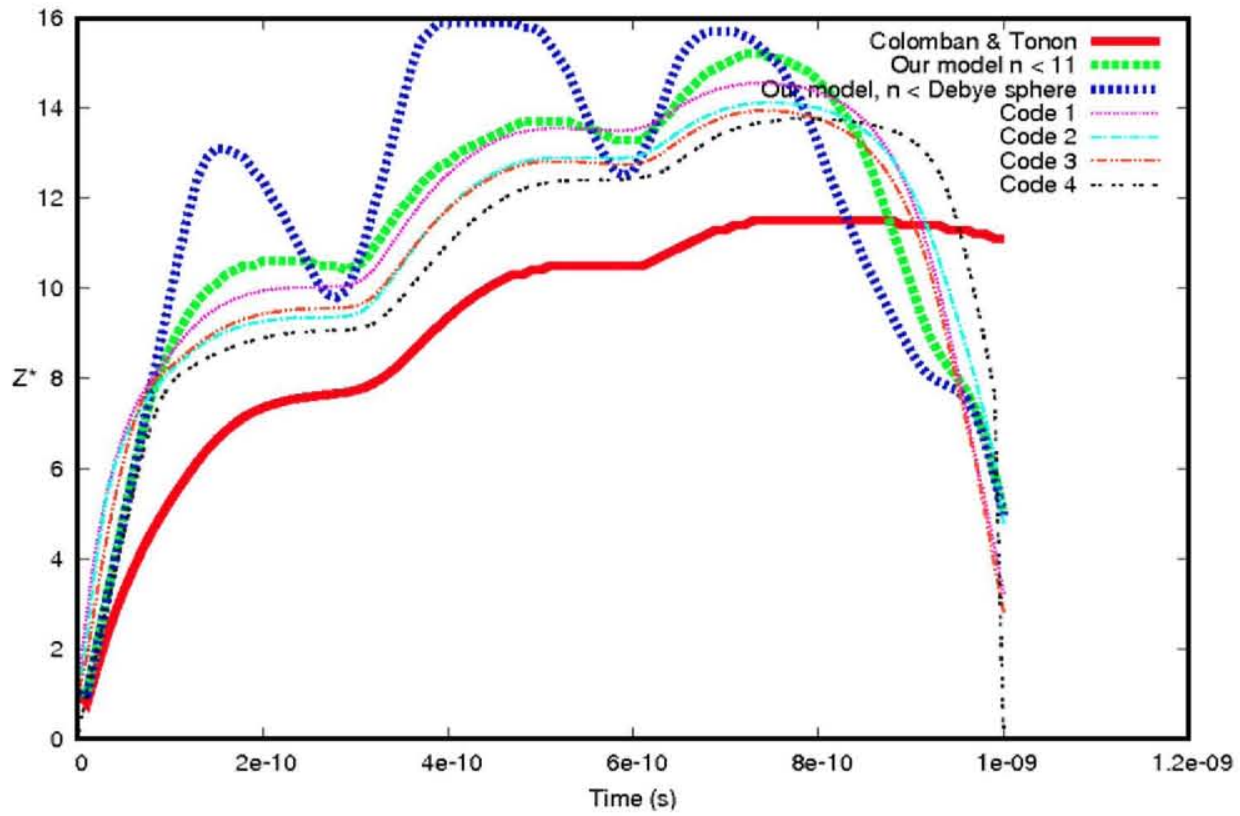

Fig. 1. Comparison of the time-dependent ionisation of Argon for various codes, with rapidly variating temperature and density taken from the NLTE4 workshop database [12]. The Colombant-Tonon model that only includes the ground level for each ion is the thick red continuous line. The fast ionisation model with $n_{\max }=10$ is the thick green dashed line. The fast ionisation model with large $n_{\max }$ only limited by the ion sphere is a thick blue dotted line. (For interpretation of the references to colour in this figure legend, the reader is referred to the web version of this article.) 
where $N_{0}$ is the population of the ground level. The global rates follow:

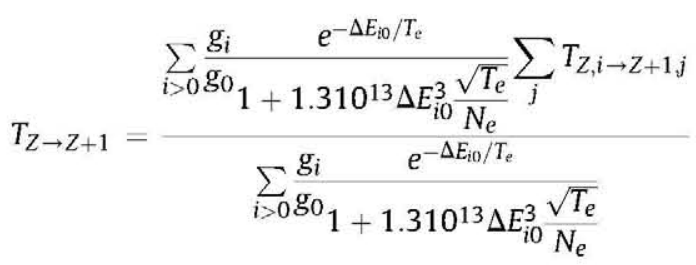

The energies of the excited levels of valence principal quantum number $n^{\prime}$ are extracted from the ionisation energy $\chi$ of the ground level $n_{0}$ :

$\chi_{\text {ionis }}\left(n^{\prime}\right)=\chi_{\text {ionis }}^{0} \frac{n_{0}^{2}}{n^{\prime 2}}$

and the degeneracies $g_{n}$ of these excited levels take into account the number of electrons $\xi$ in the valence shell:

$g_{n^{\prime}}=g_{0} \times \frac{\xi}{2 n_{0}-\xi+1} \times 2 n^{\prime 2}$

Ionisation energies and ground configurations are taken from literature [25] or computed with the FAC atomic code [26,27]. The maximum principal quantum number $n^{\prime}$ is limited to 10 . At higher densities maximum principal quantum number $n^{\prime}$ is also limited by pressure ionisation. Multiply-excited levels are not taken into account, as we assume that their contribution is less important when transient ionisation occurs. The reason is that singly-excited states tend to be directly ionised by the high energy free electrons. For this reason, autoionisation as well as dielectronic recombination are avoided in this model. For steady-state conditions this may lead to more than $10 \%$ over ionisation near closed shells in heavy elements [14]. The effective temperature of excited levels assumes that they are immediately populated. This leads to an overestimate of the ionisation rate. But this problem may be mitigated by fixing the proportion of excited states inside the ion, limiting the maximum ionisation rate between ions. The numerical scheme used to compute the variation of ionisation is the two- steps model, developed by Pert [19], which allows one to use long hydrodynamic time steps even when ionisation timescales are much shorter

Systematic comparison has been conducted at the NLTE workshops and show moderate discrepancy except at high densities where steady-state ionisation should occur. We present in Fig. 2 the difference of steady-state Molybdenum ionisation between our code and the detailed SCRIC code [28], based on FAC atomic data with 40.000 configurations. Discrepancies remain moderate except for densities higher than $10 \mathrm{mg} / \mathrm{cc}$ where discrepancies $\Delta Z>2$ occur but we assume that these conditions $\left(N_{e}>10^{21} e-/ c c\right)$ correspond to non-lasing plasmas. The previously mentioned lack of autoionisation explains this difference. The few possible comparisons for transient cases also show good agreement for low to medium densities when compared with the more refined codes as can be seen in Fig. 1. At high densities (i.e. more than $N_{e}>10^{21} e-/ c c$ ), this model shows poor agreement, but once again this not the regime where soft X-ray lasing occurs.

\subsection{Gain computation}

The gain is computed simultaneously with the ionisation by use of a quasi-steady state model with three levels. We assume that both the upper and lower lasing levels are in a steady-state with the ground level of the lasing ion. Ion populations are time dependent and therefore level populations are also. However, the ratio of excited levels to ground level is in a steady-state [29]. We rely on FAC computed collisional cross sections and radiative rates. We use a Voigt convolution of Doppler and natural plus collisional Gaussian broadening to obtain the maximum gain [30] at the centre of the lasing line:

$g_{0}=\left(N_{3}-\frac{g_{3}}{g_{2}} N_{2}\right) \frac{A_{32} \lambda_{32}^{2}}{8 \pi} \Phi_{V}\left(\nu_{0}\right)$

with

$\Phi_{V}\left(\nu_{0}\right)=\frac{1+a}{1+1.66 a+1.772 a^{2}} \frac{1}{\sqrt{\pi} \Delta \nu_{D}}$

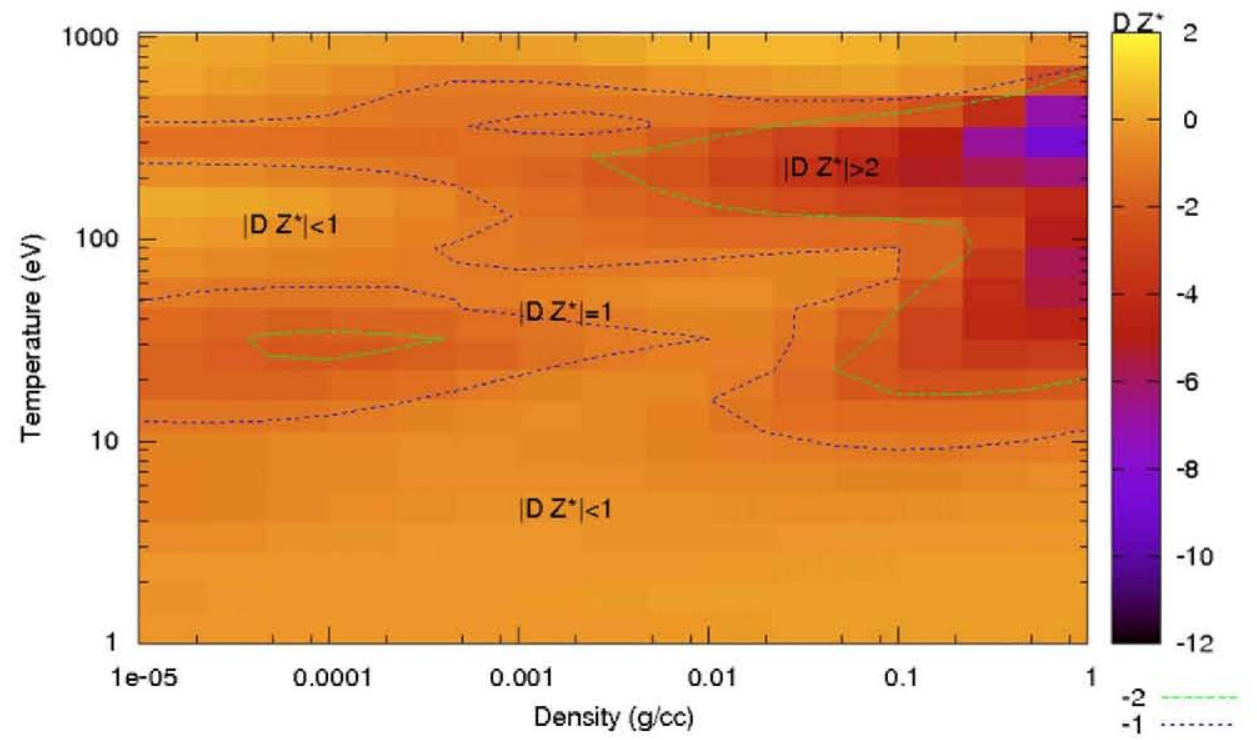

Fig. 2. Simulated differences in ionisation between the fast ionisation model and a detailed NLTE code (SCRIC [28]), for a Molybdenum plasma as a function of temperature and density. 
where $\Delta \nu_{D}$ is the Doppler broadening, $a=\Gamma_{T} / 4 \pi \Delta \nu_{D}$ and $\Gamma_{T}$ is the sum of the inverses of the lasing levels lifetimes.

If the ion temperature is not included in the hydrodynamic code, it may be inferred from the collision rate between electrons and ions:

$\nu_{e i}=3.810^{-6} \frac{Z^{*} N_{e} \ln \Lambda}{\left(k T_{e}\right)^{3 / 2}}$

and we compute :

$T_{i}(t+\Delta t)=\left(T_{i}(t)-T_{e}(t)\right)^{*} e^{-\frac{2}{3} \frac{m_{e}}{m_{i}} \nu_{e i} Z^{*} \Delta t}+T_{e}(t)$

where $\ln \Lambda$ is the Coulomb logarithm and $m_{e}$ and $m_{i}$ are the electron and ion masses.

\subsection{Effective gain and output energy}

The computation of gain with the previous formulae leads, in transient conditions, to very high gains which can reach a thousand $/ \mathrm{cm}[31,32]$. In experiments the maximum obtained never goes above $90 / \mathrm{cm}[33,34]$. The reason comes mostly from inhomogeneities and refraction in the plasma that prevents the soft X-rays from fully amplifying along the lasing plasma. Further, there is a difference between the meanings of the computed gain and the experimental gain. The computed gain is obtained as a time-dependent quantity, independent of the plasma length (i.e. it is computed for infinitely small plasma length) and presented at its maximum value (7) for the central wavelength.

$\frac{\mathrm{d} I_{v_{0}}(x)}{\mathrm{d} x}=j_{v_{0}}+g_{v_{0}} I_{v_{0}}(x) \rightarrow I_{v_{0}}(x+\mathrm{d} x) \approx I_{v_{0}}(x) e^{g_{v_{0}} \mathrm{~d} x}$

In the experimental case, the effective gain $g_{\text {eff }}$ is obtained as a variation of the total energy output $E_{\text {tot }}$ of the soft X-ray laser with the length of the plasma for finite length:
$E_{\mathrm{tot}}\left(l_{2}\right) \approx E_{\mathrm{tot}}\left(l_{1}\right) e^{g_{\mathrm{eff}}\left(l_{2}-l_{1}\right)} \rightarrow g_{\mathrm{eff}} \approx \frac{\ln \left(\frac{E_{\mathrm{tot}}\left(l_{2}\right)}{E_{\mathrm{tot}}\left(l_{1}\right)}\right)}{l_{2}-l_{1}}$

where $l_{1}$ and $l_{2}$ are the minimum possible lengths that can be measured (and are typically $1 \mathrm{~mm}$ and more)

$E_{\mathrm{tot}}=\int_{t=0}^{\infty} \int_{\nu=0}^{\infty} \int_{x} \int_{y} I_{\nu}(t, x, y) \mathrm{d} \nu \mathrm{d} t \mathrm{~d} x \mathrm{~d} y$

To compute the output intensity, frequency-integrated, we rely on the formula developed by G. Pert [35] and derived from the Linford formula to take into account the effect of saturation on the gain:

$I_{l}=I_{0} \times \alpha(X)=\frac{j_{0}}{g_{0}} \times \frac{\left(e^{X}-1\right)^{3 / 2}}{\sqrt{X e^{X}}}$

and

$\left(1-\frac{I_{0}}{I_{\text {sat }}}\right) X+\frac{I_{0}}{I_{\text {sat }}} \alpha(X)=g_{0} l$

where $I_{0}=j_{0} / g_{0}, j_{0}=N_{3} \cdot h \nu_{32}(\Omega / 4 \pi) \Phi_{V}\left(\nu_{0}\right)$ being the emissivity within the solid angle seen from the beginning of the lasing plasma and $I_{\text {sat }}$ is the saturation intensity assuming a Voigt profile.

\section{Experimental comparison}

With this model, we simulated a Ni-like Molybdenum soft X-ray laser at $18.9 \mathrm{~nm}$ currently in use at the Laserix facility (Palaiseau) [36-38]. It is created by three successive $800 \mathrm{~nm}$ laser pulses irradiating a Molybdenum solid slab of $4 \mathrm{~mm}$ length. First, a $35 \mathrm{~mJ}$ prepulse and, second, there is a $465 \mathrm{~mJ} 300$ ps FWHM heating pulse

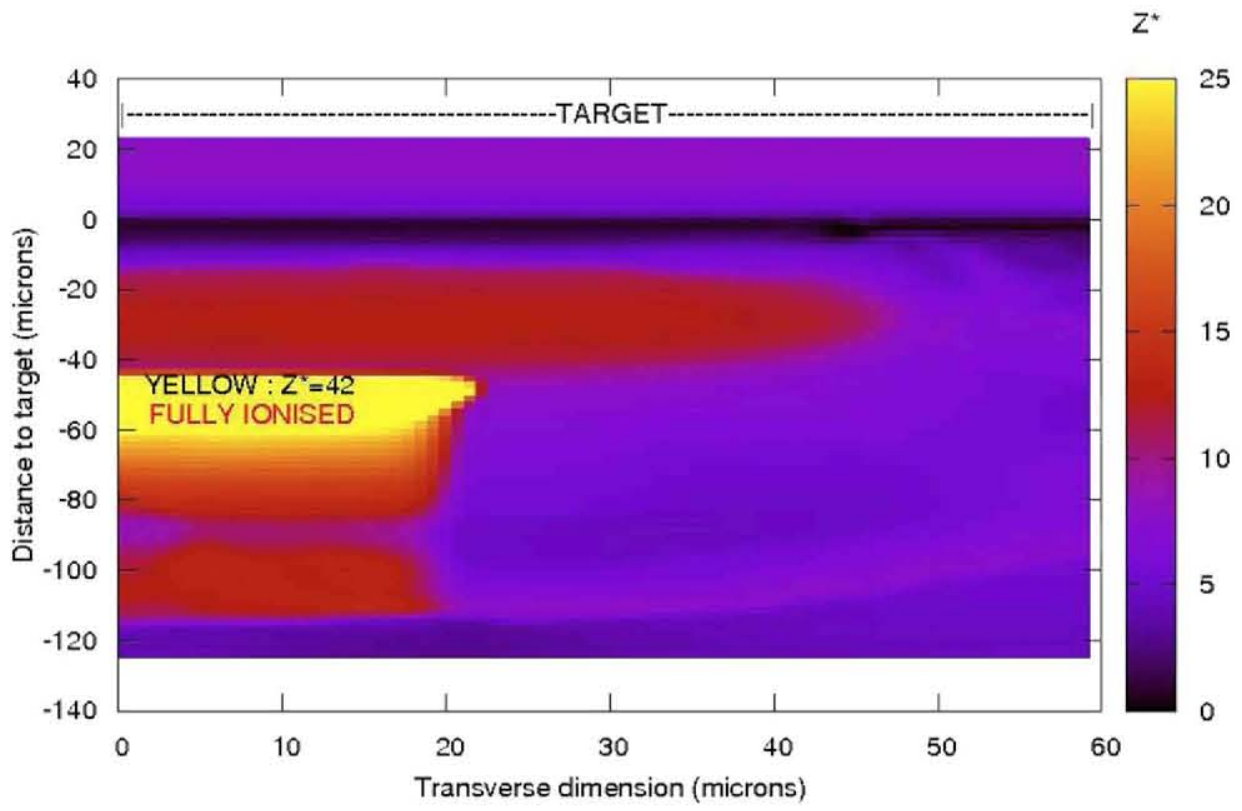

Fig. 3. Simulated transverse map of the lasing plasma ionisation computed by the ARWEN code with an LTE database, 5 ps after the peak of the short heating pulse ( 5 ps FWHM). For clarity the colour scale is limited and yellow (or white) corresponds to the highly ionised plasma. Ions are fully stripped in the yellow (or white) zone between 45 and $60 \mu \mathrm{m}$. (For interpretation of the references to colour in this figure legend, the reader is referred to the web version of this article.) 
at normal incidence focused to a $85 \mu \mathrm{m}$ FWHM width and $5 \mathrm{~mm}$ length. These two pulses are separated by $1 \mathrm{~ns}$. They are followed by a $500 \mathrm{~mJ}, 5 \mathrm{ps}$ Grazing Incidence (GRIP) of $40 \mu \mathrm{m}$ width, incident at $19^{\circ}$ from the slab surface, which arrives $0.7 \mathrm{~ns}$ after the second pulse. The incident pulse is simulated by changing the wavelength from $800 \mathrm{~nm}$ to $2.46 \mu \mathrm{m}$ to have a turning point consistent with the new critical density. We are aware that this is a simplification and may lead to an over deposition near the critical density as can be inferred from Ref. [39].

We model this experiment in three different ways: 1) using the ARWEN code with LTE integrated ionisation followed by a postprocessing with our fast ionisation model, 2) using the hydrodynamic subroutine of the ARWEN code with our fast ionisation model embedded and finally, 3) using our fast ionisation model alone for various final temperatures and densities.

\subsection{Postprocess of an LTE hydrodynamic simulation}

To speed up computations, the ARWEN code uses precomputed LTE ionisation tables. We present in Fig. 3 the ionisation obtained in the code with the LTE tables just at the end of the fast heating pulse. One can see that the plasma is fully ionised in the interaction zone. If we postprocess the hydrodynamics with our NLTE steady-state routine (not shown), it also results in fully stripped ions over a broad but smaller area at the end of the short pulse.

We have post-processed the hydrodynamics data obtained from the previous simulation with the fast ionisation routine conserving only the electron temperature and the ion density. The increase in ionisation is shown in Fig. 4 and shows a lower average ionisation than in the LTE/NLTE steady-state case. Whereas the lasing ion is $14+$, the ionisation decreases below 12.5 , yielding very low population of the lasing ion. After 2400 ps, which is 400 ps after the GRIP pulse, the temperature decreases (not shown) from $2000 \mathrm{eV}$ at its maximum in the interacting zone to lower than $60 \mathrm{eV}$. The gain, in Fig. 5, reaches its maximum at 2050 ps. As saturation is usually obtained for gain-length products larger or equal to 16 , we assume that the $4 \mathrm{~mm}$ long laser is saturated for gains larger or equal to $40 \mathrm{~cm}^{-1}$. The saturation in these conditions lasts $63 \mathrm{ps}$. We present in Table 1 a comparison with experimental results which shows a substantial overestimate of the duration and gain. The duration of the soft X-ray laser had been broadly estimated to be 5 ps which is consistent with resolved time-measurements as in Ref.[40].)

\subsection{Hydrodynamic simulation with fast ionisation included}

A new version of the hydrodynamic module of the ARWEN code has been written to include the fast ionisation routine. It is not yet coupled with the other modules of laser absorption, electron conduction and radiative transfer. For this reason we used as input the results of the previous LTE hydrodynamic simulation at the time of the GRIP pulse. The ionisation balance and ion density just before the GRIP pulse are used and assumed to be constant during the GRIP pulse. We keep the energy absorbed at the end of the GRIP pulse but with the constant ionisation that leads to new electronic temperatures. Then the system relaxes with this new hydrodynamic routine including fast ionisation.

In this post-processing, the initial maximum temperature is much higher in the laser-plasma interacting zone than in the previous case, $12 \mathrm{keV}$ as compared to $2000 \mathrm{eV}$, but the ionisation increases slowly as can be seen in Fig. 6 . It takes 60 ps to increase from $12+$ to $16+$ and after 1 ns the ionisation reaches $30+$ in the high temperature zone. For this reason the temperature remains much higher during the whole simulation and this leads to much higher maximum gains reaching $470 / \mathrm{cm}$ as seen in Table 1 and lasting much longer as seen in the same table and in Fig. 7. However, such temperatures can not remain for so long, as conduction effects propagate the heat away limiting the temperature while radiative losses, which are proportional to the fourth power of the temperature, will also reduce the temperature.

\subsection{Fast ionisation model alone}

The reason for the discrepancy between our previous results and the experiment comes from various biases in the simulation: lack of conduction, simplicity of the ionisation model and the

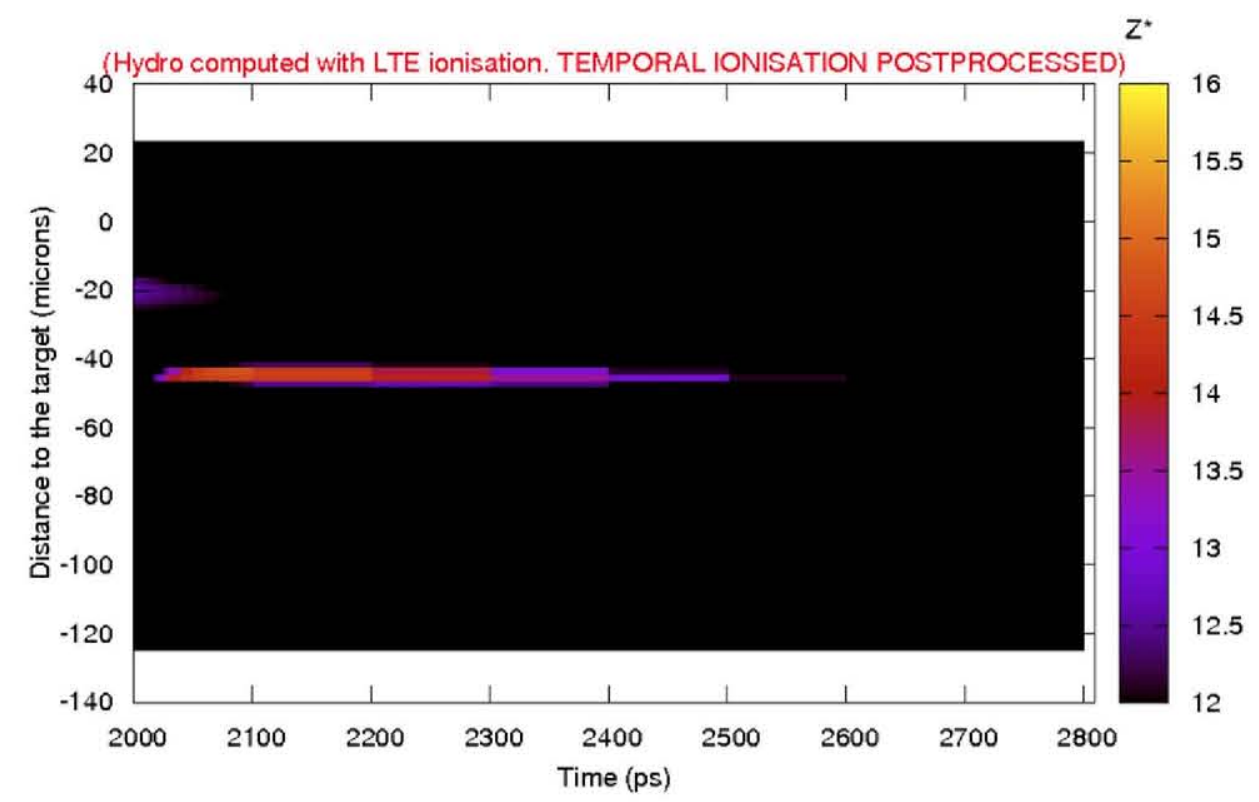

Fig. 4. Simulated time-dependent ionisation post-processed after an LTE based simulation with ARWEN as function of distance from the target and time. For clarity and compare with Fig. 6 , ionisation is only presented between $12+$ and $16+$ where lasing may occur. 


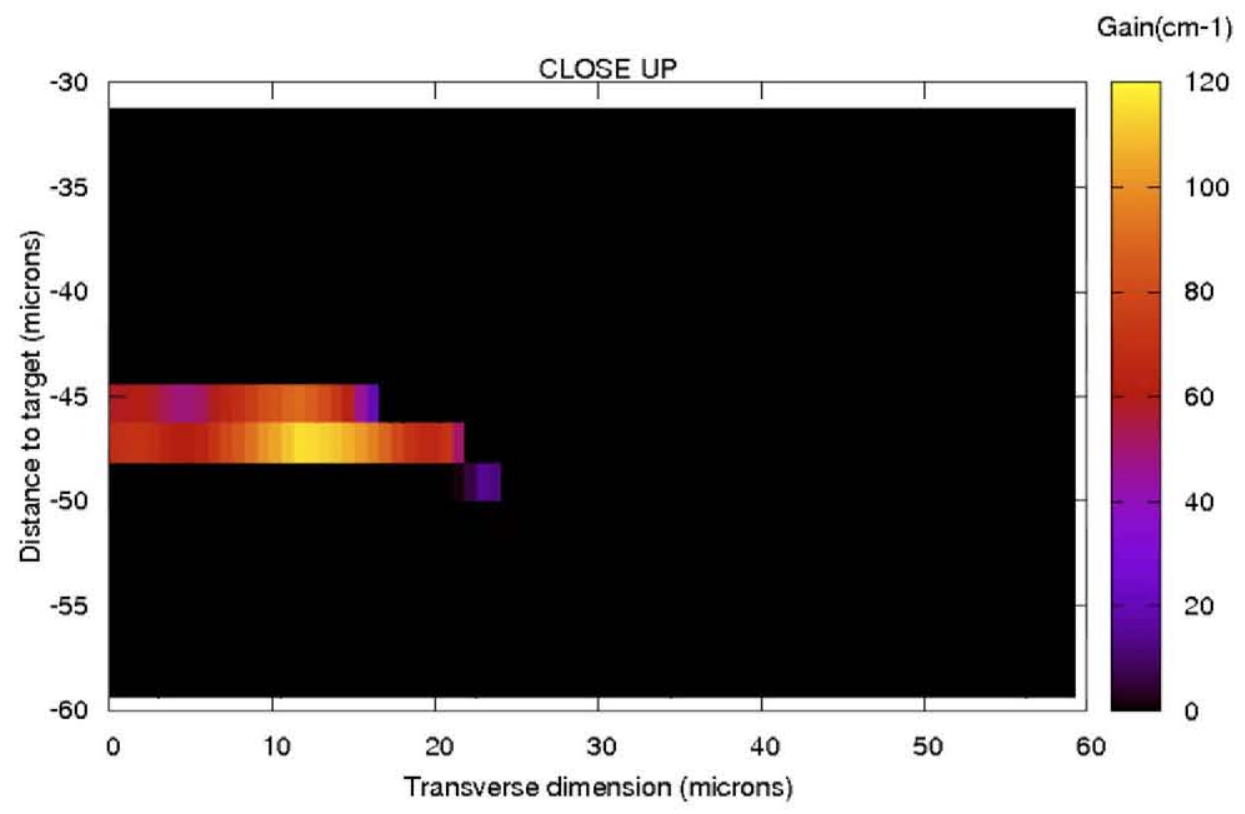

Fig. 5. Simulated transverse map of the lasing plasma gain post-processed with the fast ionisation routine after an ARWEN simulation with an LTE database. Gain is presented when it reaches its maximum, 50 ps after the peak of the GRIP pulse ( 5 ps FWHM). The target is located at $+20 \mu \mathrm{m}$.

quasi-steady-state populations of lasing levels. The modification of the GRIP pulse by changing the wavelength may explain the four to five times difference in thickness of the lasing plasma (the width in the direction perpendicular to the target surface) with the experiment presented in Table 1. A thicker interaction zone would also strongly limit the temperature of the lasing zone. Moreover, there are differences with the manner in which the experiment and the simulations determine the lasing output. In the experiment, the results are time-, space- and frequencyintegrated, while the simulations results are space- and timedependent values at the lasing fixed-frequency, as explained in Sec. 2.3.

We present in Fig. 8 the effects of longitudinal integration on gain, duration and intensity of a time-integrated laser emission of a Molybdenum plasma. To simulate the soft X-ray laser, we choose a density of $2.2810^{20} \mathrm{e}-/ \mathrm{cc}$ which corresponds to the critical density. The temperature of $1400 \mathrm{eV}$ corresponds to the energy of the laser after absorption in the interaction volume of $25 \mu \mathrm{m} \times 40 \mu \mathrm{m} \times 6 \mathrm{~mm}$ with $50 \%$ losses. Tallents [41] assumes $30 \%$ of the energy of the laser beam is lost to nonlinear effects and we assume that radiative losses may also limit the energy of the electrons. This seems consistent with temperatures found in other simulations conducted on Molybdenum and Palladium with an appropriate GRIP absorption [39].

The gain curve with respect to the plasma length in Fig. 8 shows the difficulty in comparing experimental and computed gain. As saturation appears to begin for very short lengths $(0.3 \mathrm{~mm})$ the gain will be experimentally measurable only in already partly saturated plasmas. For a measure between 0.5 and $1 \mathrm{~mm}$, the energy increase leads to a gain of $35 / \mathrm{cm}$ in broad agreement with the experimental gain of $40 / \mathrm{cm}$. But if measured between 1 and $2 \mathrm{~mm}$, it leads to $11 / \mathrm{cm}$ much smaller than that measured. Nevertheless, this is not comparable with the maximum time-dependent gain obtained during the simulation: $997 / \mathrm{cm}$. We also note that the duration of the laser depends on its length. 1) For very small lengths, the emission of the plasma with time is directly proportional to the time-dependent gain, therefore the duration of the laser is equivalent to the duration of the gain. 2) When the gain length product increases, the intensity increases as $\exp (3 g l / 2)$. The duration of the laser is of the order of the full-width at half-maximum of the timedependent intensity. The half-maximum intensity is obtained when the gain reaches $g_{I_{\max } / 2}=g_{I_{\max }}-(2 \ln 2 / 3 l)$. This means that the minimum gain to reach the half-maximum intensity increases with length. Therefore the time for which the gain is high enough to have larger intensities than half-maximum intensity decreases with length. 3) When the gain length product becomes large enough to reach the saturation intensity, the intensity increases very little when the gain increases. The time duration of the laser becomes equivalent to the duration over which saturation is reached. For this reason a minimum in duration for the soft X-ray laser is reached for lengths where the time-dependent intensity reaches saturation and the duration increases with length.

We present in Fig. 9 the duration of the soft X-ray laser pulse computed for various densities and final electron temperatures.

Table 1

Comparison of experimental results of a Molybdenum soft X-ray laser [36] with hydro-radiative simulations with time-dependent ionisation either post-processed or inline and with the fast ionisation routine alone. When the fast ionisation routine is used alone, density is $2.28 \mathrm{mg} / \mathrm{cc}$ and the suddenly imposed temperature is $1400 \mathrm{eV}$.

\begin{tabular}{llrr}
\hline & Transient ionisation post-processed (ARWEN complete) & Transient ionisation inline (hydro alone) & Fast ionisation model alone \\
\hline Gain $\left(\mathrm{cm}^{-1}\right)$ & 130 & 470 & $35-11$ \\
Gain duration $(\mathrm{ps})$ & 63 & 191 & 40 \\
Distance to target $(\mu \mathrm{m})$ & 42.5 & 39.5 & 5 \\
Thickness $(\mu \mathrm{m})$ & $2-4$ & 5.9 & 35 \\
Energy $(\mu \mathrm{J})$ & & & 25 \\
\hline
\end{tabular}




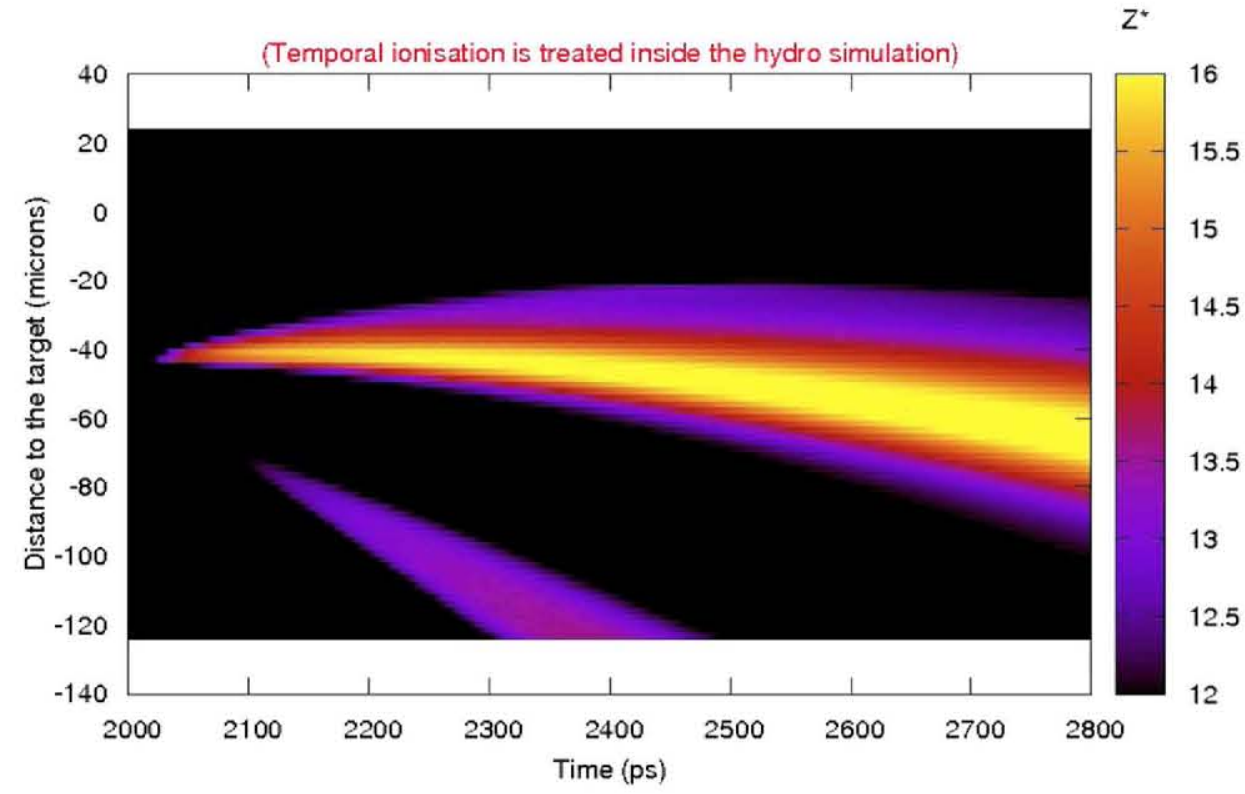

Fig. 6. Simulated time-dependant ionisation obtained inline with the hydrodynamic module of the ARWEN code as function of distance from the target and time. For better reading and compare with Fig. 4 , ionisation is only presented between $12+$ and $16+$ where lasing may occur.

Our fast ionisation routine code allows us to compute the duration, final energy and gain in minutes on a desk computer. This gives one access to the approximate parameters that can optimize the lasing before using more refined models to test the possible cases. In biology, possible applications of soft X-ray lasers require short pulse durations. We see in our computation that this is obtained by increasing the density of the plasma but is less dependent on the temperature. Our comparison with experiment can be seen in Fig. 8 and Table 1. It shows that we overestimate the duration by a factor of 2 or 3 . The reason may be due to low ionisation rates as we only included in our calculation the valence shell ionisation $n_{1}^{p_{1}} \ldots n_{m}^{p_{m}} n^{*} \rightarrow n_{1}^{p_{1}} \ldots n_{m}^{p_{m}}$ whereas $n_{1}^{p_{1}} \ldots n_{m}^{p_{m}} n^{*} \rightarrow n_{1}^{p_{1}} \ldots$ $n_{m}^{p_{m}-1} n^{*}$ may also be important, especially for initially closed shells in which the number of electrons compensate for the higher ionisation energies.

The output energy of the soft X-ray laser (Table 1) appears to be larger by a factor 2.8 to 14 than found in the experiment, depending on the width of the soft X-ray laser focus chosen: $5 \mu \mathrm{m}$ as in the simulation results as compared to $25 \mu \mathrm{m}$ in the experiment. Apart from the temporal, spatial and spectral averaging mentioned in Eq. (12), detectors integrate the radiation over a much broader bandwidth than the soft X-ray laser, thus including in the output energy incoherent emission of the plasma. For this reason the experimental energy suffers an uncertainty of a factor 2 . The long duration of our modelled laser gain might also account for a factor of 2 or 3 .

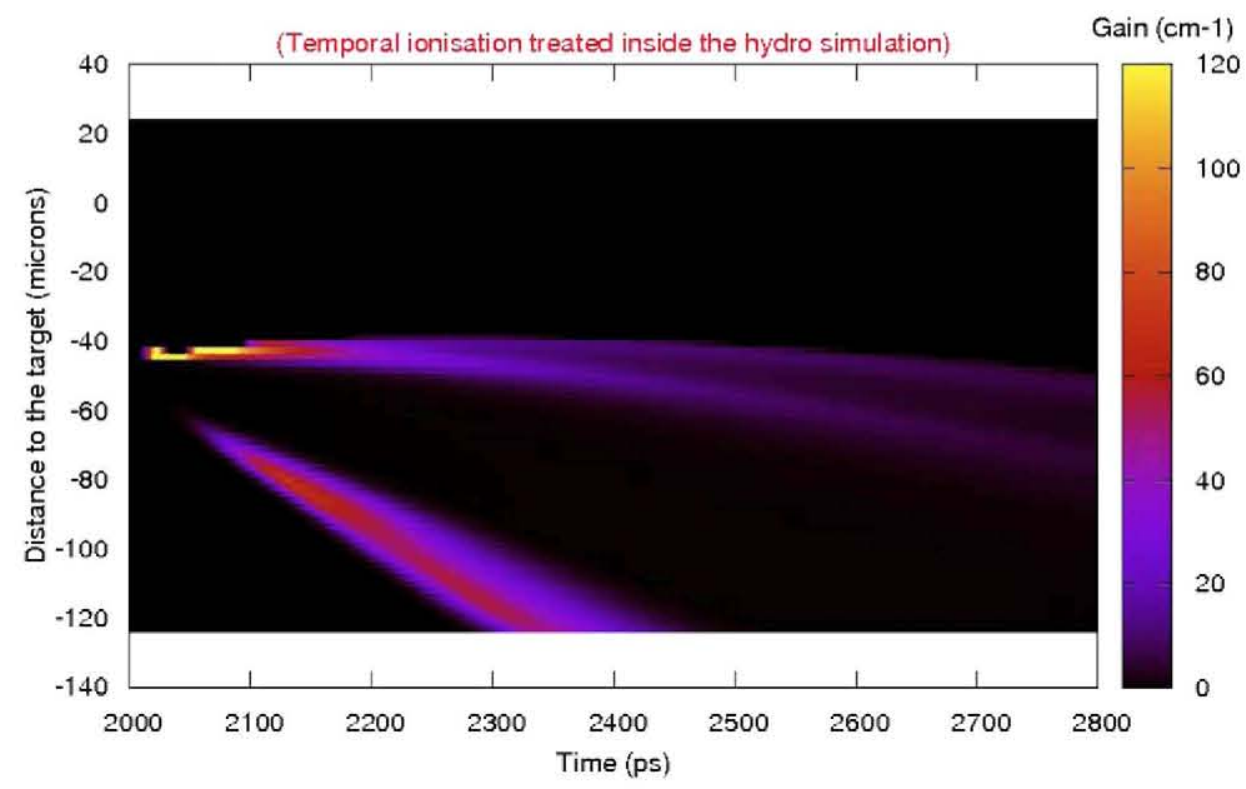

Fig. 7. Simulated time-dependant gain obtained inline with the hydrodynamic module of the ARWEN code as function of distance from the target and time. 


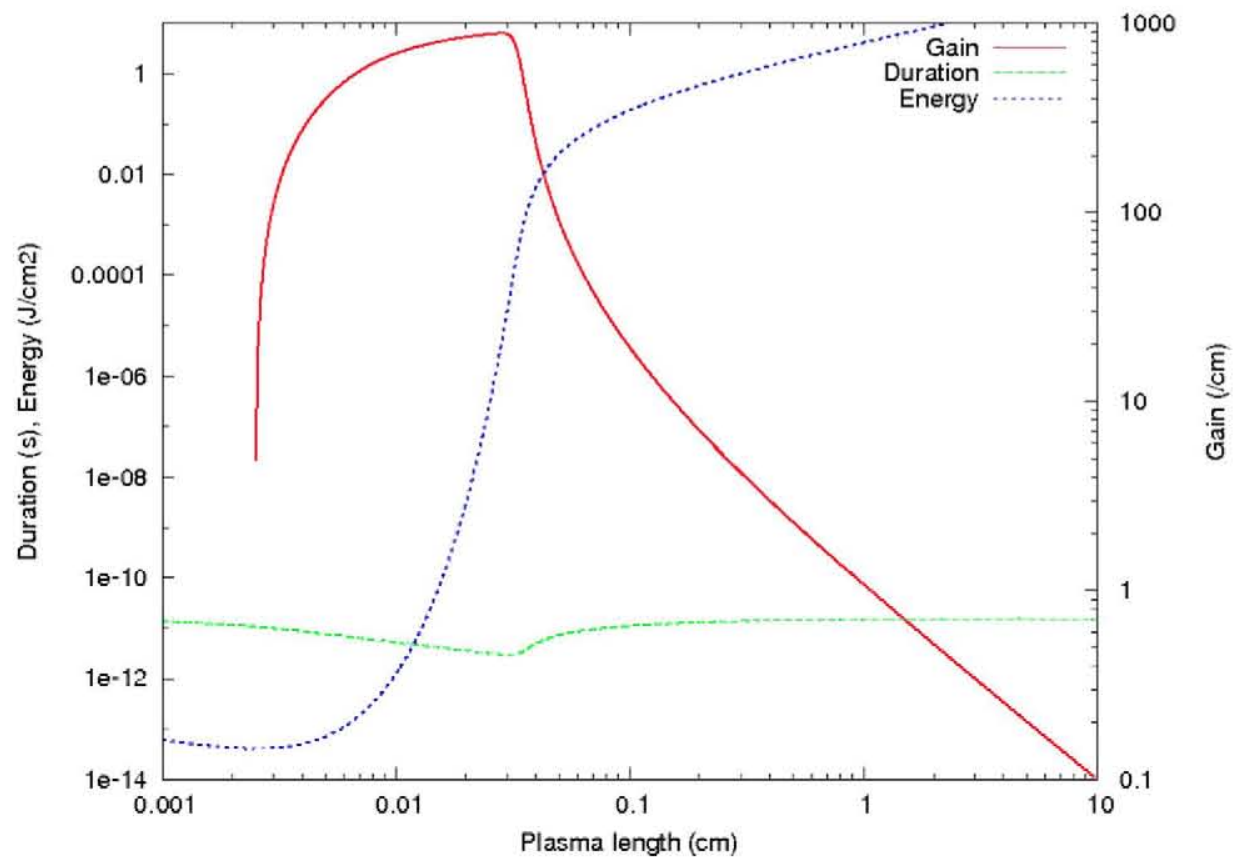

Fig. 8. Simulated variation of the time-integrated gain, duration and energy output as function of plasma length of a Molybdenum Ni-like lasing plasma at 2.28 mg/cc created by suddenly increasing the temperature to $1400 \mathrm{eV}$.

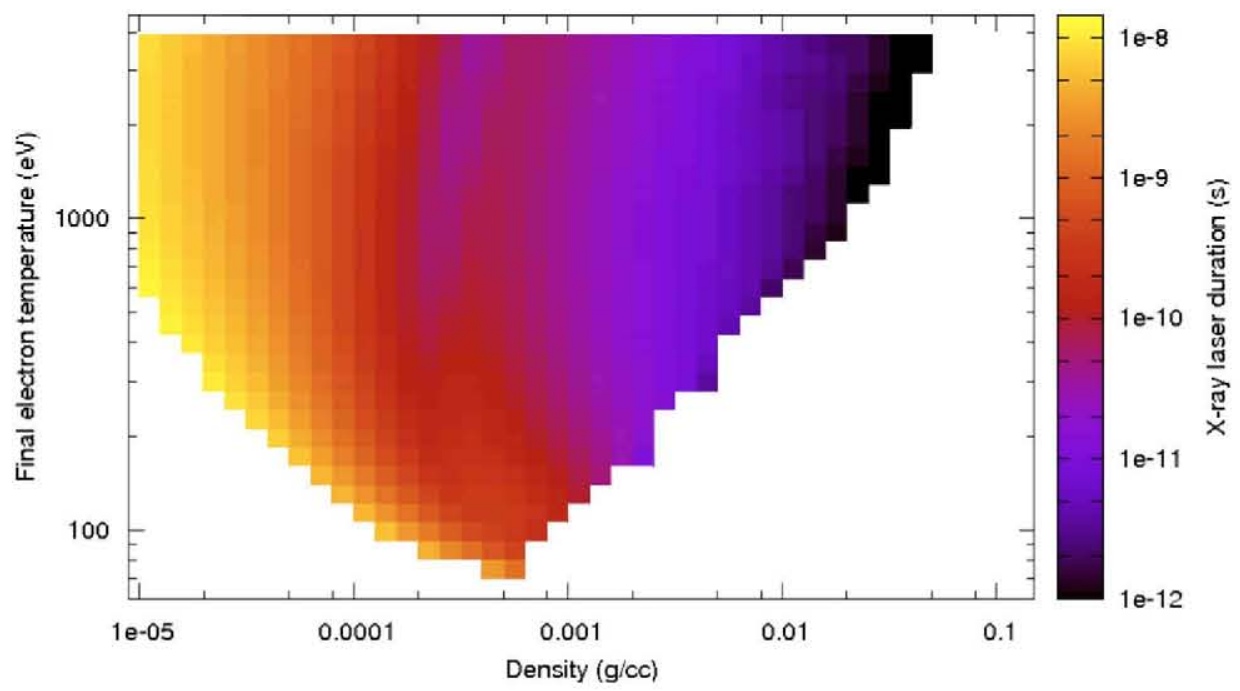

Fig. 9. Simulated duration of a $4 \mathrm{~mm}$ long lasing plasma with the fast ionisation model as function of density and suddenly imposed temperature.

\section{Summary and conclusions}

The simulations presented here show convergence with some aspects of the experimental results. The fast ionisation part alone leads to stationary ionisations with 5-10\% difference compared to more refined but much slower ionisation codes. In transient cases, ionisation rates and gain are found within a factor of three of more detailed simulations and experiments. Inclusion of the fast ionisation routine within the hydrodynamic routine of ARWEN alone leads to a substantial modification of the temperature and ionisation when compared to the LTE data-based simulations - even when post-processed with the fast ionisation model. The comparison with a more realistic fully integrated fast ionisation model including also conduction, radiative transfer and realistic laser absorption in addition to hydrodynamics is currently being developed.

\section{Acknowledgements}

The authors would like to thank S. Hansen, G. Pert and G. Tallents for their explanations and help and acknowledge the fruitful European COST MP0601 action and the financial support provided by the Spanish Ministerio de Educación y Cultura program no. ENE2009-09837 and to the European Commission projects HiPERPP (211737) and SFINX-LASERLAB-II.

\section{References}

[1] G.B. Zimmerman, W.L. Kruer, Comments Plasma Phys. Contr. Fusion 2 (1975) 51.

[2] R. Ramis, et al., Comp. Phys. Comm. 49 (1988) 475.

[3] S. Jacquemot, A. Decoster, Laser Part. Beams 9 (1991) 517.

[4] J.P. Christiansen, D.E.T.F. Ashby, K.V. Roberts, Comput. Phys. Commun. 7 (1974) 271.

[5] F. Ogando, P. Velarde, J. Quant. Spectrosc. Radiat. Transf. 71 (2001) 541.

[6] J. Filevich, J.J. Rocca, et al., Phys. Rev. E 67 (2003) 056409. 
[7] E. Oliva, P. Zeitoun, S. Sebban, M. Fajardo, P. Velarde, K. Cassou, D. Ros, Opt. Lett. 34 (2009) 17

[8] A.G. de la Varga, et al., High Energy Density Phys, this volume (2011)

[9] A.G. de la Varga, BIGBART. Relativistic Screened Hydrogenic Code for Radiation Hydrodynamics, Technical Report. Instituto de Fusión Nuclear, 2010.

[10] H.K. Chung, R.W. Lee, High Energy Density Phys. 5 (2009) 1-14.

[11] C. Bowen, R.W. Lee, Y. Ralchenko, JQSRT 99 (2006) 102

[12] J.G. Rubiano, R. Florido, C. Bowen, Y. Ralchenko, High Energy Density Phys. 3 (2007) 225.

[13] C.J. Fontes, J. Abdullah Jr., C. Bowen, R.W. Lee, Y. Ralchenko, High Energy Density Phys. 5 (2009) 15.

[14] Y. Ralchenko, et al., High Energy Density Phys., this volume (2011).

[15] J. Abdullah Jr., M.E. Sherrill, High Energy Density Phys. 4 (2008) 124-130.

[16] H.K. Chung, M. Chen, W. Morgan, Y. Ralchenko, R. Lee, High Energy Density Phys. 1 (2005) 3-12.

[17] H. Scott, S.B. Hansen, High Energy Density Phys. 6 (2010) 39-47.

[18] G. Pert, J. Fluid Mech. 131 (1983) 401-426.

[19] G. Pert, J. Comput. Phys. 27 (1978) 241

20] D. Colombant, G.F. Tonon, J. Appl. Phys. 44 (1978) 3524.

[21] M. Poirier, et al., JQSRT 99 (2006) 482-492.

[22] J. White, et al., J. Appl. Phys. 101 (2007) 043301.

[23] R. Rakowski, et al., Appl. Phys. B. 101 (2010) 773-789.

[24] S. Hansen, et al., JQSRT 99 (2006) 272.
[25] R.D. Cowan, The Theory of Atomic Structure and Spectra. University of California Press, Berkeley, 1980

[26] M.F. Gu, Astrophys. J. 582 (2003) 1241.

[27] M.F. Gu, Can. J. Phys. 86 (2008) 251.

28] F. de Gaufridy, SCRIC, Technical Report No 6115. CEA, 2006

[29] G. Pert, Phys. Rev. A 73 (2006) 033809.

[30] K.G. Withney, A. Dasgupta, P.E. Pulsifer, Phys. Rev. E 50 (1994) 468-473.

[31] A. Sasaki, et al., JQSRT 71 (2001) 665.

[32] R.F. Smith, et al., Phys. Rev. E 72 (2005) 036404

33] P.V. Nickles, K.A. Janulewicz (Eds.), X-ray Lasers 2006, Springer, 2007.

[34] C.L.S. Lewis, D. Riley (Eds.), X-ray Lasers 2008, Springer, 2009.

[35] G. Pert, J. Opt. Soc. Am. B. 11 (1994) 4412

[36] S. Kazamias, K. Cassou, D. Ros, F. Plé, G. Jamelot, A. Klisnick, O. Lundh, F. Lindau, A. Persson, C.G. Wahlström, S. de Rossi, D. Joyeux, B. Zielbauler, D. Ursescu, T. Kühl, Phys. Rev. A 77 (2008) 033812.

[37] K. Cassou, S. Kazamias, D. Ros, F. Plé, G. Jamelot, A. Klisnick, O. Lundh, F. Lindau, A. Persson, C.-G. Wahlström, S. de Rossi, D. Joyeux, B. Zielbauer, D. Ursescu, T. Kühl, Opt. Lett. 32 (2007) 139.

[38] B. Zielbauer, D. Zimmer, J. Habib, O. Guilbaud, S. Kazamias, M. Pittman, D. Ros, Appl. Phys. B. 100 (2010) 731.

[39] F. Yan, et al., Phys. Rev. A 73 (2006) 023812.

[40] Y. Ochi, et al., Appl. Phys. B. 78 (2004) 961.

[41] G.J. Tallents, J. Phys. D 36 (2003) R259-R276. 